\title{
CERTAIN UNIFORM FUNCTIONS OF RATIONAL FUNCTICNS*
}

BY

EMORY P. STARKE

I. INTRODUCTION

A natural generalization of the notion of an even function (i.e., a uniform function of $z^{2}$ ) is that of a uniform function of any rational function of $z$. In this paper, we examine the following classes of functions and determine which functions in each class are uniform functions of non-linear rational functions :

(1) periodic meromorphic functions;

(2) Poincare's functions with rational multiplication theorems. $\dagger$

Among the periodic functions, we shall find in §III that only the following possess the desired property:

(i) Meromorphic functions of $\cos (\lambda z+\mu)$. These are uniform functions of $(z+\mu / \lambda)^{2}$.

(ii) Rational functions of $\wp(z+\mu)$. These are uniform functions of $(z+\mu)^{2}$.

(iii) Rational functions of $\rho^{2}(z+\mu)$ in the lemniscatic case $\left(g_{8}=0\right)$. These are functions of $(z+\mu)^{4}$.

(iv) Rational functions of $q^{\prime}(z+\mu)$ or of $q^{3}(z+\mu)$ in the equianharmonic case $\left(g_{2}=0\right)$. These are functions of $(z+\mu)^{3}$ or of $(z+\mu)^{6}$ respectively.

In the above $\lambda$ and $\mu$ are arbitrary constants.

For the Poincare functions ( $\$ I V)$, our results are as follows:

(a) If in any Poincare function we replace $z$ with $z^{q}$, where $q$ is an integer, we shall have another Poincare function which is a uniform function of $\boldsymbol{z}^{\boldsymbol{q}}$.

(b) Every Poincaré function which is a uniform function of a non-linear rational function and which is not uniform in any $z^{q}$, is a periodic function. Using the results of a paper by Ritt, we see that such functions are necessarily linear functions of one of the functions $\cos (\lambda z+\mu), \wp(z+\mu)$; in the lemniscatic case, $\wp^{2}(z+\mu)$; in the equianharmonic case, $\wp^{\prime}(z+\mu)$ and $\wp^{3}(z+\mu)$. Here $\mu$ is restricted to certain values.

* Presented to the Society, May 1, 1926; received by the editors in May, 1926.

† These are described in detail below (\$IV). 


\section{UNIFORM FUNCTIONS OF RATIONAL FUNCTIONS: SOME GENERAL RESULTS}

Let $f(z)$ be a non-constant, meromorphic function satisfying a relation (1)

$$
f(z)=g[\sigma(z)],
$$

with $\sigma(z)$ rational and not linear, and with $g(z)$ uniform. Without loss of generality, we may require $g(z)$ to be also meromorphic; for, since $f(z)$ is meromorphic, $g(z)$ cannot have other essential singularities than $\sigma(\infty)$. We may suppose, replacing $\sigma(z)$ by a linear function of itself if necessary, that $\sigma(\infty)=\infty$. Having now no finite essential singularity, $g(z)$ is meromorphic.

$\sigma(z)$ then is a polynomial; for, if it had a pole at a finite point, this point would be an essential singularity for the meromorphic $f(z)$. We may now put (multiplying $\sigma(z)$ by a constant if necessary to give unity for the leading coefficient)

$$
\sigma(z)=z^{n}+b_{1} z^{n-1}+\cdots+b_{n} .
$$

The $n$ branches of the inverse of $u=\sigma(z)$ have, for the neighborhood of infinity, the single expansion

$$
z=\sigma^{-1}(u)=u^{1 / n}+c_{0}+c_{1} u^{-1 / n}+\cdots .
$$

Consider one of the branches $z$ in (3), and the branch $z^{\prime}$ by which it is replaced when $u$ describes a single circuit about a large circle in the positive direction. We are going to express $z^{\prime}$ in terms of $z$.

For a single circuit of $u, u^{1 / n}$ goes over into $\epsilon u^{1 / n}$ where $\epsilon=e^{2 \pi i / n}$. If then in (3) we replace $u^{1 / n}$ by $\epsilon u^{1 / n}$ and substitute for $u^{1 / n}$ that branch of $[\sigma(z)]^{1 / n}$ which is used in the calculation of the original branch $z$ in (3), we shall have $z^{\prime}$ expressed as a function of $z$. Writing $z^{\prime}=\alpha(z)$, we find thus, for $|z|$ sufficiently large,

$$
\alpha(z)=\epsilon z+a_{0}+\sum_{n=1}^{\infty} a_{n} z^{-n} .
$$

The principal part of the proofs in both of the following sections will be to show that in the cases there treated $\alpha(z)$ is linear. Let us assume then that $\alpha(z)$ is not linear, and fix our attention on the first non-vanishing coefficient of the summation in (4). If its subscript is $p$, equation (4) now takes the form

$$
\alpha(z)=\epsilon z+a_{0}+a_{p} z^{-p}+\cdots, \quad a_{p} \neq 0, \quad p \geqq 1 .
$$


We shall use also the inverse of $\alpha(z)$, which, like $\alpha(z)$, is analytic for $|z|$ large. Its development for the neighborhood of infinity is, if $p>1$,

$$
\begin{aligned}
\alpha^{-1}(z)= & \frac{z-a_{0}}{\epsilon}-\frac{\epsilon^{p-1} a_{p}}{z^{p}}-\frac{\epsilon^{p-1}\left(p a_{0} a_{p}+\epsilon a_{p+1}\right)}{z^{p+1}} \\
& -\frac{\epsilon^{p-1}\left[\frac{1}{2}\left(p^{2}+p\right) a_{0}^{2} a_{p}+(p+1) \epsilon a_{0} a_{p+1}+\epsilon^{2} a_{p+2}\right]}{z^{p+2}}+\cdots .
\end{aligned}
$$

If $p=1$, the term $+\epsilon a_{1}^{2}$ should be added to the expression in brackets. But this peculiarity of the case $p=1$ does not carry over into the calculations of the following sections; we secure the same equations for $p>1$ and for $p=1$.

Of course, for $|z|$ large, we have

$$
\sigma(z)=\sigma[\alpha(z)]=\sigma\left[\alpha^{-1}(z)\right],
$$

and thus

$$
f(z)=f[\alpha(z)]=f\left[\alpha^{-1}(z)\right] .
$$

\section{Periodic functions}

In addition to the hypotheses of the foregoing section, let $f(z)$ be periodic, either simply or doubly, and let $h$ be any period of $f(z)$. We take any finite point $z$, of large modulus, and proceed to find certain other points at which $f(z)$ assumes the same value as at $z$. We form the expressions

(7a) $z_{1}=\alpha(z)+h=\epsilon z+a_{0}+h+a_{p} z^{-p}+a_{p+1} z^{-p-1}+a_{p+2} z^{-p-2}+\cdots$,

(7b) $z_{2}=\alpha^{-1}\left(z_{1}\right)+h=z+h+\frac{h}{\epsilon}+\frac{p a_{p} h}{\epsilon^{2} z^{p+1}}$

$$
+\frac{2 \epsilon(p+1) a_{p+1} h-p(p+1) a_{p} h^{2}}{2 \epsilon^{8} z^{p+2}}+\cdots,
$$

(7c) $\quad z_{z}=\alpha\left(z_{2}\right)-h=\epsilon z+a_{0}+\epsilon h+\frac{a_{p}}{z^{p}}+\frac{a_{p+1}}{z^{p+1}}+\frac{a_{p+2}}{z^{p+2}}-\frac{p a_{p} h}{z^{p+1}}$

$$
+\frac{(p+1)\left[p a_{p} h^{2}(2+\epsilon)-2 \epsilon h a_{p+1}\right]}{2 \epsilon z^{p+2}}+\cdots
$$

Putting $\phi(z)=\alpha^{-1}\left(z_{z}\right)-h$, we find

$$
\phi(z)=z+\frac{p(p+1) h^{2} a_{p}}{\epsilon^{2} z^{p+2}}+\cdots .
$$


In view of (6) and of the relation $f(z \pm h)=f(z)$, we have

$$
f[\phi(z)]=f\left(z_{i}\right)=f(z) \quad(j=1,2,3) .
$$

If $|z|$ is sufficiently large, there will be a $z_{1}$, a $z_{2}$, a $z_{3}$, and a $\phi(z)$ which will satisfy (9).

To fix our ideas, suppose that $|z+h| \geqq|z|$. Consider the points $z+j h$ $(j=1,2, \cdots)$. (If $|z+h|<|z|$, take the points $z-j h$, instead.) For all these points, the relation (9) holds. If now, according to (8), we form the expression,

$$
\phi(z+j h)-j h=z+\frac{p(p+1) h^{2} a_{p}}{\epsilon^{2}(z+j h)^{p+2}}+\cdots,
$$

we see that the left-hand member gives, as $j$ assumes all positive integral values, a set of points of which infinitely many are in every neighborhood of $z$, but for each of which the function $f(z)$ takes the same value as at $z$. Since $z$ is not an essential singular point of the meromorphic function $f(z)$, we have a contradiction. The assumption that $\phi(z)$ is not linear, which we made in $\left(4^{\prime}\right)$, is therefore untenable. We have then

$$
\alpha(z)=\epsilon z+a_{0} .
$$

If we differentiate the relation

$$
\sigma(z)=\sigma[\alpha(z)]=\sigma\left(\epsilon z+a_{0}\right)
$$

$s$ times, we obtain the equation

$$
\sigma^{(\bullet)}(z)=\epsilon^{*} \sigma^{(\bullet)}\left(\epsilon z+a_{0}\right) .
$$

Noting that $\epsilon\left[a_{0} /(1-\epsilon)\right]+a_{0}=a_{0} /(1-\epsilon)$, and that $\epsilon^{*} \neq 1$ for $s=1,2, \cdots$, $n-1$, we see that the first $n-1$ derivatives of $\sigma(z)$ must vanish for the value $z=a_{0} /(1-\epsilon)$. The polynomial $\sigma(z)$ has therefore the form

$$
\sigma(z)=\left[z-\frac{a_{0}}{1-\epsilon}\right]^{n}+k,
$$

in which $k$ is a constant. Since we may still add to $\sigma(z)$ an arbitrary constant if necessary, we put

$$
\sigma(z)=(z-c)^{n}, \quad c=a_{0} /(1-\epsilon) .
$$

Referring to equation (7b) and remembering that now $a_{p}=0, a_{p+1}=0$, $\cdots$, we see that $h / \epsilon$ is a period of $f(z)$. As $h$ is any period, it follows that $h$ multiplied by any power of $\epsilon$ will be a period. Since, further, $\sigma(z)$ is not linear, we must have $n>1$ and $\epsilon \neq 1$. 
If $f(z)$ is simply periodic, with periods $h$ and $h / \epsilon$, evidently $\epsilon$ must be -1 and $n$ must be 2 . We have

$$
\begin{array}{cc}
\alpha(z)=2 c-z, & \sigma(z)=(z-c)^{2}, \\
f(z)=f(2 c-z)= & g\left[(z-c)^{2}\right] .
\end{array}
$$

If we write $c+h u / 2 \pi$ for $z, c^{\prime} h / 2 \pi$ for $c$ and $F(2 \pi z / h)$ for $f(z)$, the relation $f(z)=f(2 c-z)$ becomes

$$
F\left(c^{\prime}+u\right)=F\left(c^{\prime}-u\right),
$$

where $F(u)$ has the period $2 \pi$. Being also even with respect to $u, F\left(c^{\prime}+u\right)$ must be a uniform function of $\cos u$. Replacing the original letters, we have $f(z)$ expressed as a meromorphic function of $\cos 2 \pi(z-c) / h$. With a slight change of notation, this gives

$$
f(z)=\psi[\cos (\lambda z+\mu)]=g\left[(z+\mu / \lambda)^{2}\right],
$$

with $\psi(z)$ and $g(z)$ meromorphic. Any meromorphic $\psi(z)$ will give an $f(z)$ which is uniform in $(z+\mu / \lambda)^{2}$.

If now $f(z)$ is doubly periodic, it is of course a rational function of $q(z)$ and $\varphi^{\prime}(z)$. It may be shown that $n$ must be 2 unless $g_{3}=0$ or $g_{2}=0$. If $g_{3}=0$, $n$ may be 2 or 4 , while if $g_{2}=0, n$ may be 2,3 , or 6 . $^{*}$

First consider the case $n=2$. We have $f(z)=f(2 c-z)$. If we set $z=u+c$, this becomes $f(c+u)=f(c-u)$. If the periods of $f(z)$ are $2 \omega_{1}$ and $2 \omega_{3}, f(c+u)$, being even with respect to $u$, is a rational function of $8\left(u / \omega_{1}, \omega_{3}\right)$ alone. We then have

$$
f(z)=\psi[\wp(z-c)]=g\left[(z-c)^{2}\right]
$$

with $\psi(z)$ rational.

If now $n=3$, we have $f(z)=f[\alpha(z)]$ or $f(z)=f[\epsilon z+(1-\epsilon) c]$. Setting $z=u+c$, we find that $f(u+c)=f(\epsilon u+c)$. Now in the equianharmonic case, $\rho^{\prime}(\epsilon u)=\varphi^{\prime}(u)$. Then both $f(u+c)$ and $\varphi^{\prime}(u)$ remain unchanged if $u$ is replaced by $\epsilon u$. Hence in the development of either function at the origin, the exponents of $u$ are all multiples of 3 . Since both functions are uniform functions of $u^{3}$, while $\varphi^{\prime}(u)$ is of the third order, we see that $f(u+c)$ is a rational function of $\varepsilon^{\prime}(u)$. We have then

$$
f(z)=\psi\left[\wp^{\prime}(z-c)\right]=g\left[(z-c)^{3}\right],
$$

with $\psi(z)$ again rational. p. 20.

* See Ritt, Periodic functions with a multiplication theorem, these Transactions, vol. 23 (1922), 
We handle the remaining cases in precisely the same manner, obtaining the following results: For $n=4$ (lemniscatic case),

$$
f(z)=\psi\left[q^{2}(z-c)\right]=g\left[(z-c)^{4}\right] .
$$

For $n=6$ (equianharmonic case),

$$
f(z)=\psi\left[p^{3}(z-c)\right]=g\left[(z-c)^{8}\right] .
$$

In (15) and (16), $\psi(z)$ is rational.

\section{The Poincaré functions}

Let $f(z)$ be a non-constant, meromorphic function of $z$ such that $f(m z)$ is a rational function of $f(z)$,

$$
f(m z)=R[f(z)],
$$

where $m$ is any constant of modulus greater than unity. Functions $f(z)$ of this type formed the object of an investigation by Poincaré.* We shall investigate the circumstances under which $f(z)$ can be put in the form,

$$
f(z)=g[\sigma(z)],
$$

in which $g(z)$ is meromorphic and $\sigma(z)$ is a non-linear polynomial as in \&II.

We indicate here a few properties of the functions $f(z)$ and $R(z)$ which will be useful in the following discussion. $\dagger$

(i) We may assume, without loss of generality, upon replacing each of the functions $f(z)$ and $R(z)$, if necessary, by a suitable linear function of itself, that $f(0)=0$ and $R(0)=0$.

(ii) If $R(z)$ is linear, $f(z)$ is a linear function of some $z^{q}$ ( $q$ an integer). $f(z)$ has then a zero of order $q$ at the origin.

(iii) If $f(z)$ has a zero of order $q$ at the origin, $f(z)$ is of the form $g\left(z^{q}\right)$, in which $g(z)$ is a Poincare function with a simple zero at the origin, and satisfies the relation

$$
g(M z)=R[g(z)], \quad M=m^{q}=R^{\prime}(0) .
$$

(iv) If $g(z)$ is any Poincare function, the function $f(z)=g\left(z^{a}\right)$ clearly satisfies both relations (17) and (18). These functions, $g\left(z^{q}\right)$, are among the functions we seek. This gives result (a) of the introduction.

* H. Poincaré, Sur une classe nouvelle de transcendantes uniformes, Journal de Mathématiques, (4), vol. 55 (1890).

$\dagger$ Noted by Ritt, Transcendental transcendency of certain functions of Poincare, Mathematische Annalen, vol. 95 (1926). 
(v) If $R(z)$ is not linear, $f(z)$ assumes the value zero at an infinite number of points.

In what follows, we set aside the functions described in (iii) and (iv) above. There remain to be considered only Poincare functions, $f(z)$, for which we have $f(0)=0, f^{\prime}(0) \neq 0$, with $R(z)$ a non-linear rational function such that $R(0)=0, R^{\prime}(0)=m$.

From equation (6), §II, we have, for $|z|$ sufficiently large,

$$
f[\alpha(z)]=f(z), \quad f[\alpha(m z)]=f(m z) .
$$

Here again, we desire to show that $\alpha(z)$ is linear. Let us then assume, as in $\left(4^{\prime}\right)$, that it is not, but contains a term $a_{p} z^{-p} \neq 0, p \geqq 1$. Using (17), we may rewrite the second of the relations (19) thus:

$$
R\{f[\beta(z)]\}=R\left\{f\left[\frac{\alpha(m z)}{m}\right]\right\}=R\{f(z)\},
$$

in which, for simplicity of notation, we have put

$$
\beta(z)=[\alpha(m z)] / m .
$$

The calculation of $\beta(z)$ and its inverse in terms of the coefficients of $\left(4^{\prime}\right)$ and (5) gives, for $|z|$ large,

$$
\begin{aligned}
\beta(z) & =\epsilon z+\frac{a_{0}}{m}+\frac{a_{p}}{m^{p+1} z^{p}}+\frac{a_{p+1}}{m^{p+2} z^{p+1}}+\cdots, \\
\beta^{-1}(z) & =\frac{z}{\epsilon}-\frac{a_{0}}{m \epsilon}-\frac{\epsilon^{p-1} a_{p}}{m^{p+1} z^{p}}-\frac{\epsilon^{p-1}\left(p a_{0} a_{p}+\epsilon a_{p+1}\right)}{m^{p+2} z^{p+1}}+\cdots
\end{aligned}
$$

We take now a point $z$ of sufficiently large modulus and seek, by combining $\alpha(z), \beta(z)$ and their inverses, to obtain a point close to $z$, at which the function $R[f(z)]$ has the same value as at $z$. We have*

$$
\begin{gathered}
\alpha^{2}(z)=\epsilon^{2} z+(\epsilon+1) a_{0}+\frac{a_{p}\left(\epsilon^{p+1}+1\right)}{\epsilon^{p} z^{p}}+\frac{a_{p+1}\left(\epsilon^{p+2}+1\right)-p a_{0} a_{p}}{\epsilon^{p+1} z^{p+1}}+\cdots, \\
\beta^{-1} \alpha^{2}(z)=\epsilon z+a_{0}\left(\frac{m \epsilon+m-1}{m \epsilon}\right)+\frac{a_{p}}{z^{p}}\left(\frac{m^{p+1} \epsilon^{p+1}+m^{p+1}-1}{m^{p+1} \epsilon^{p+1}}\right) \\
+\frac{1}{z^{p+1}}\left[a_{p+1}\left(\frac{m^{p+2} \epsilon^{p+2}+m^{p+2}-1}{m^{p+2} \epsilon^{p+2}}\right)-p a_{0} a_{p}\left(\frac{\epsilon m^{p+2}-\epsilon m-m+1}{m^{p+2} \epsilon^{p+3}}\right)\right]+\cdots,
\end{gathered}
$$

\footnotetext{
- We abbreviate $\beta^{-1}\{\alpha[\alpha(z)]\}$ thus: $\beta^{-1} \alpha^{2}(z)$. Similarly for the other functions.
} 


$$
\begin{aligned}
\alpha^{-1} \beta^{-1} \alpha^{2}(z)=z & +\frac{a_{0}(m-1)}{m \epsilon^{2}}+\frac{a_{p}\left(m^{p+1}-1\right)}{m^{p+1} \epsilon^{p+2} z^{p}} \\
& +\frac{1}{z^{p+1}}\left[a_{p+1}\left\{\frac{\left(m^{p+2}-1\right)}{m^{p+2} \epsilon^{p+3}}\right\}\right. \\
& \left.+p a_{0} a_{p}\left\{\frac{\left(\epsilon^{p+1} m^{p+1}+1\right)(m-1)-\epsilon m\left(m^{p+1}-1\right)}{m^{p+2} \epsilon^{p+4}}\right\}\right]+\cdots
\end{aligned}
$$

Similarly, we have

$$
\begin{aligned}
\beta^{-1} \alpha^{-1} \beta^{2}(z)=z & -\frac{a_{0}(m-1)}{m \epsilon^{2}}-\frac{a_{p}\left(m^{p+1}-1\right)}{m^{p+1} \epsilon^{p+2} z^{p}} \\
& -\frac{1}{z^{p+1}}\left[a_{p+1}\left\{\frac{\left(m^{p+2}-1\right)}{m^{p+2} \epsilon^{p+3}}\right\}\right. \\
& \left.+p a_{0} a_{p}\left\{\frac{\left(\epsilon^{p+1}+m^{p+1}\right)(m-1)-\epsilon\left(m^{p+1}-1\right)}{m^{p+2} \epsilon^{p+4}}\right\}\right]+\cdots
\end{aligned}
$$

We now form the combination

$$
\phi(z)=\beta^{-1} \alpha^{-1} \beta^{2} \alpha^{-1} \beta^{-1} \alpha^{2}(z) .
$$

This gives

$$
\phi(z)=z+\frac{0}{z^{p}}+\frac{p a_{0} a_{p}(m-1)\left(m^{p+1}-1\right)\left(\epsilon^{p}-1\right)}{m^{p+2} \epsilon^{p+3} z^{p+1}}+\cdots
$$

Since we had

$$
R\{f[\alpha(z)]\}=R\{f(z)\}=R\{f[\beta(z)]\}
$$

and

$$
R\left\{f\left[\alpha^{-1}(z)\right]\right\}=R\{f(z)\}=R\left\{f\left[\beta^{-1}(z)\right]\right\},
$$

we shall have also

$$
R\{f[\phi(z)]\}=R\{f(z)\} .
$$

We put $d_{k}$ for the coefficient of $z^{-k}$ in (22), so that (22) becomes

$$
\phi(z)=z+d_{p} z^{-p}+d_{p+1} z^{-p-1}+d_{p+2^{-p-2}}+\cdots
$$

If $|z|$ is large enough there will be a point $\phi(z)$ given by (24) which will satisfy the relation (23). But we shall show that $\phi(z)$, as given by (24), approximates too closely to $z$ for (23) to hold, unless $\phi(z)=z$.

Suppose that $\phi(z)$ is not identically $z$. Let $\gamma$ be a point of large modulus for which we have $f(\gamma)=0$. Evidently, we shall have also $f\left(m^{\circ} \gamma\right)=0$, for any integer $s$. 
We consider, in addition, the point $\zeta=\phi\left(m^{\imath} \gamma\right) / m^{2}$, for which we have the relation

$$
R^{s+1}[f(\zeta)]=R\left[f\left\{\phi\left(m^{s} \gamma\right)\right\}\right]=R\left[f\left(m^{s} \gamma\right)\right]=R^{s+1}[f(\gamma)]=0 .
$$

$R(z)$ has a development about the origin of the form

$$
R(z)=m z+\cdots,
$$$$
|m|>1 \text {. }
$$

There exists a positive number $\rho$ such that, in (26), we have

$$
\left|m^{1 / 2} z\right|<|R(z)|<\left|m^{3 / 2} z\right|, \text { for } 0<|z| \leqq \rho .
$$
form

Since $f(\gamma)=0$, we can develop $f(z)$, for the neighborhood of $\gamma$, in the

$$
f(z)=A_{j}(z-\gamma)^{j}+A_{i+1}(z-\gamma)^{i+1}+\cdots
$$

Using (24), we find

$$
\zeta=\frac{\phi\left(m^{2} \gamma\right)}{m^{\bullet}}=\gamma+\frac{d_{p}}{m^{\bullet(p+1)} \gamma^{p}}+\frac{d_{p+1}}{m^{2(p+2)} \gamma^{p+1}}+\cdots
$$

For $s$ large, $\zeta \neq \gamma$, so that $f(\zeta) \neq 00^{*}$ For sufficiently large values of $s$, we may substitute the result of (29) into (28), and obtain

$$
f(\zeta)=\frac{A_{j} d_{p}^{i}}{m^{i<(p+1)} \gamma^{i p}}+\frac{j A_{j} d_{p}^{i-1} d_{p+1}}{m^{i(p i+j+1)} \gamma^{p i+1}}+\cdots
$$

The absolute value of the denominator of the first term in (30) is not less than $\left|m^{2} s\right|$. Hence, for $s$ large, we have

$$
|f(\zeta)|<\rho /\left|m^{3 s / 2}\right|<\rho .
$$

From (27) and (31) we obtain, for $s$ large,

$$
\left|m^{1 / 2} f(\zeta)\right|<|R[f(\zeta)]|<\left|m^{3 / 2} f(\zeta)\right|<\left|\rho / m^{3(0-1) / 2}\right| .
$$

We may therefore put $R[f(\zeta)]$ for $z$ in $(27)$, and have

$$
\left|m^{1 / 2} R[f(\zeta)]\right|<\left|R^{2}[f(\zeta)]\right|<\left|m^{3 / 2} R[f(\zeta)]\right| \text {. }
$$

But this gives

$$
|m f(\zeta)|<\left|R^{2}[f(\zeta)]\right|<\left|\rho / m^{3(s-2) / 2}\right|<\rho .
$$

Continuing in this way, we find

$$
\left|m^{2 / 2} f(\zeta)\right|<\left|R^{t}[f(\zeta)]\right|<\left|\rho / m^{3(s-t) / 2}\right|<\rho \quad(t=1,2, \cdots, s),
$$

\footnotetext{
- It is understood in this that $\gamma$ is first chosen and held fixed, and that sufficiently large values are then given to $s$.
} 
and finally,

$$
\left|m^{(s+1) / 2} f(\zeta)\right|<\left|R^{s+1}[f(\zeta)]\right|<\rho|m|^{3 / 2} \text {. }
$$

The contradiction between (25) and (32) shows that $\phi(z)$ must be identically $z$. The coefficients $d$ in (24) are thus zero. In particular we have

$$
d_{p+1}=p a_{0} a_{p}(m-1)\left(m^{p+1}-1\right)\left(\epsilon^{p}-1\right) /\left(m^{p+2} \epsilon^{p+3}\right)=0 .
$$

Recalling that $p \geqq 1,|m|>1$ and that $a_{p}$ is assumed to be different from zero, we have either $a_{0}=0$ or $\epsilon^{p}=1$.

We see that $a_{0}$ cannot be zero from the following considerations. Suppose $a_{0}=0$, and form from $\left(4^{\prime}\right)$ and (21) the function

$$
\alpha \beta^{-1}(z)=z+\frac{\left(m^{p+1}-1\right) \epsilon^{p} a_{p}}{m^{p+1} z^{p}}+\cdots
$$

Now we have $R\left\{f\left[\alpha \beta^{-1}(z)\right]\right\}=R\{f(z)\}$. If we represent $\alpha \beta^{-1}(z)$ by $\phi(z)$, the discussion above from equations (23) to (32) will hold for this new $\phi(z)$. We are led to the result $a_{p}=0$, which is supposed not to be true. Hence $a_{0} \neq 0$.

On the other hand, $\epsilon^{p}$ cannot be 1. To show this, we expand the polynomial $\sigma(z)=\sigma[\alpha(z)]$ in the form

$$
\begin{aligned}
\sigma(z)=\sigma\left(\epsilon z+a_{0}\right)+\sigma^{\prime}\left(\epsilon z+a_{0}\right) & {\left[\frac{a_{p}}{z^{p}}+\frac{a_{p+1}}{z^{p+1}}+\cdots\right] } \\
& +\frac{\sigma^{\prime \prime}\left(\epsilon z+a_{0}\right)}{2 !}\left[\frac{a_{p}}{z^{p}}+\frac{a_{p+1}}{z^{p+1}}+\cdots\right]^{2}+\cdots
\end{aligned}
$$

If now $p \geqq n$, there is in (33) only one term of degree $(n-1-p)$. Its coefficient, $n \epsilon^{n-1} a_{p}$, however, cannot be zero. Hence $p$ must be less than $n$. But if $\epsilon^{p}=1$, since $\epsilon=e^{2 \pi i / n}$, we must have $p$ a multiple of $n$. It follows that $\epsilon^{p} \neq 1$.

The contradiction noted in equation (32) cannot be removed under our assumption of the existence of an $a_{p} \neq 0$. Hence $\alpha(z)$ is linear, and we have, as in \$III, the relations

$$
\alpha(z)=\epsilon z+a_{0}, \quad \sigma(z)=(z-c)^{n}, \quad a_{0}=(1-\epsilon) c .
$$

So then we have $f(z)=f\left(\epsilon z+a_{0}\right)$, and also

$$
f(z)=R^{s}\left[f\left(z / m^{s}\right)\right]=R^{s}\left[f\left(\epsilon z / m^{e}+a_{0}\right)\right]=f\left(\epsilon z+m^{s} a_{0}\right) .
$$

We obtain thus the relation

$$
f\left(\epsilon z+a_{0}\right)=f\left(\epsilon z+m^{s} a_{0}\right) .
$$


Here too, $a_{0}$ cannot be zero. For, if $f(z)=f(\epsilon z)$, it would follow that $f(z)$ is a meromorphic function of $z^{n}$, and $f^{\prime}(0)$ is zero. But such functions have previously been set aside.

Equation (34) then recites the fact that $f(z)$ is a periodic function with $\left(m^{2}-1\right) a_{0}$, a period. This establishes result (b) of the introduction: every Poincare function which is not uniform in any $z^{q}$, and which satisfies a relation (18), is a periodic function.

Periodic functions which satisfy (18) are listed in §III. Periodic functions which satisfy (17) are given in a paper by Ritt.* The comparison of these results shows that periodic Poincare functions which are uniform functions of non-linear rational functions are linear functions of one of the functions $\cos (\lambda z+\mu), \quad(z+\mu) ; p^{2}(z+\mu)$ if $g_{3}=0 ; p^{\prime}(z+\mu)$ and $p^{3}(z+\mu)$ if $g_{2}=0$. Here $\mu$ is an appropriate fraction of a period.

* Ritt, Periodic functions with a multiplication theorem, these Transactions, vol. 23 (1922), pp. 16-25.

Columbia University,

NEW York, N. Y. 